\title{
A Comparison of an Actuarial and a Linear Model for Predicting Organizational Behavior
}

\author{
Blake A. Frank \\ The Southland Corporation
}

Using an actuarial and a linear model for predicting organizational behavior, employee subgroups were identified through a hierarchical and convergent clustering of assessment variable profiles in a validation sample $(N=2,899)$ and cross-validated by assigning a holdout sample $(N=2,899)$ to the original subgroups on the basis of a minimum distance qualifier. Subgroup membership in both samples was significantly associated with current employment status and job performance. A linear discriminant function analysis of employment status and a linear regression analysis of job performance also yielded significant results. A comparison of the two models in terms of predictive accuracy indicated that the two models were essentially equivalent. However, it was concluded that the actuarial model was superior to the linear model, since a descriptive and behavioral taxonomy based on stable, homogeneous employee subgroups could be developed.

In recent years there have been two broad criticisms of the traditional linear model as applied to the prediction of behavior in organizations. The first criticism holds that the traditional model is inadequate to predict complex organizational behavior because it does not account for configurations of individuals and their unique interactions with task demands and the organizational environment (Dunnette, 1963). A

APPLIED PSYCHOLOGICAL MEASUREMENT

Vol. 4, No. 2 Spring 1980 pp. 171-181

(C) Copyright 1980 West Publishing Co. similar sentiment was expressed by Tyler (1959), who criticized the generally low ceiling on predictability achieved by the traditional model and suggested a new approach based on patterns of choice behavior. The second criticism concerns the inability of the traditional linear model to contribute very much to the understanding of human behavior (Owens, 1971; Tyler, 1959). Since the efficacy of the linear model is empirically determined, little or no causal attribution can be made for observed relationships between variables.

The foundation for a departure from the traditional model was developed by Toops (1948, 1959), who believed that society consisted of an array of identifiable, homogeneous subgroups of individuals and that, once identified, these subgroups could be used to predict the future behavior of subgroup members. In essence, Toops described a model for the actuarial prediction of behavior. Actuarial prediction refers to procedures that involve the derivation of probability estimates of future behavior from contingentfrequency tables (Wiggins, 1973). The actuarial prediction problem takes the following form: Given several subgroups $\left(G_{1}, G_{2}, \ldots, G_{k}\right)$, what is the probability that an individual in a particular group will be a member of a given criterion category $\left(C_{1}, C_{2}, \ldots, C_{j}\right)$ ? Sines $(1966)$ referred to this strategy as prediction from taxonomic classes. 
Recent research on the actuarial model has shown that homogeneous subgroups can be identified from heterogeneous groups of individuals and that these subgroups are significantly related to external criteria (Pinder \& Pinto, 1974; Pinto, 1970; Schoenfeldt, 1974; Taylor, 1968). Additionally, the utility of using such subgroups as moderators in least squares procedures, probability weighting methods, and a Bayesian regression model has been researched (Lissitz \& Schoenfeldt, 1974). In this study subgroup membership was input into several different prediction models, but in each case variables other than those used to define the subgroups were also used in the development of each prediction model.

No research comparing the actuarial and linear models appears to have been conducted in which the same set of variables on the one hand, has been used to define the subgroups from which predictions are made and, on the other hand, has been used as the independent variables in the development of a least squares prediction equation. The purpose of this study was to compare an actuarial prediction model with the linear model, both developed on an identical set of variables in the same sample, for effectiveness in prediction. The research consisted of the following four phases: (1) development of homogeneous subgroups from a heterogeneous pool of exempt-level employees; (2) validation and cross-validation of predictions made from subgroup membership (i.e., the actuarial model); (3) validation and cross-validation of predictions made from the linear model; and (4) comparison of the actuarial and linear models for effectiveness in predicting relevant organizational criteria.

\section{Method}

\section{Subjects}

The subjects were 5,798 exempt-level employees of a large petrochemical company. The sample consisted of all individuals who had been tested in the company's ongoing managerial assessment program and who were employed, or had been employed, in one of five broad work force categories: upper level management $(8.2 \%)$, supervisory professional-technical $(10.5 \%)$, supervisory professional $(8.4 \%)$, professional-technical (36.4\%), and professional (36.6\%).

The sample was randomly divided into validation and cross-validation samples of 2,899 employees each. Chi-square and $t$ tests indicated no significant differences between the samples on any of the demographic variables (e.g., age, tenure) or analysis variables available for study.

\section{Criteria}

Two criteria relevant to organizational success were developed. First, in light of a rather substantial attrition rate (33\%), employment status was selected as one criterion. On the basis of data in their personnel files, employees in both the validation and cross-validation samples were assigned to either a "With Company/Retired" category or a "Not With Company" category. The former category contained individuals who were currently employed by the company or who had worked at the company until retirement; the latter category contained individuals who had either left the company or had been terminated by the company between the time they completed the managerial assessment test battery and the date of data collection for this research.

Second, prior to splitting the sample, a job performance measure was developed from a principal components analysis of age, company service, job grade, performance appraisal, and an estimate of career potential (Frank, 1976; Vicino \& Bass, 1978). Factor scores were computed on a factor defined by high positive loadings on job grade (.76), performance appraisal (.84), and potential estimate (.94), and low loadings on tenure (.11) and age (-.10). High- and low-criterion groups were obtained by dichotomizing the factor scores at the mean of each sample. 


\section{Analysis Variables}

All scores or subscores available from the firm's managerial assessment test battery were used as analysis variables. Complete data on the following 13 scores were available for all employees:

1. Developmental Influences: The nature of early experiences in the home;

2. Achievement-Academic Years: The level of formal education and achievements while in school;

3. Present Self-Concept: Self-image and level of confidence;

4. Staff Communication/Participation: Philosophy regarding communication with one's staff and participation in decision making;

5. Employee Selection/Development: Philosophy regarding the methods and criteria for selecting and developing employees;

6. Employee Motivation/Labor Relations: Beliefs regarding the methods for rewarding employees and the responsibility of the organization to its employees;

7. Management Style/Decision Making: Orientation of managers to superiors and subordinates and ability to make decisions;

8. Behavioral Consistency: Consistency of temperament and amount of self-control;

9. Energy Level/Time Use: Pace of work and time utilization;

10. Confidence/Conviction: The confidence placed in one's own ideas and plans;

11. Behavior Understanding/Tolerance: Conflict proneness and tolerance of others' beliefs and ideas;

12. Verbal Reasoning: As measured by the Miller Analogies Test (Psychological Corporation, 1970);

13. Nonverbal Reasoning: As measured by the Test of Nonverbal Reasoning (Richardson, Bellows, Henry, \& Co., 1963).

The first three variables were developed from a biographical information blank. Scores 4 through 7 were derived from a test of managerial judgment, and Scores 8 through 11 were developed from a temperament survey. These measures are subscores from the Manager Profile Record, an empirically keyed instrument designed to predict management potential (Richardson, Bellows, Henry, \& Co., 1973).

\section{Actuarial Model}

Subgroup development. Employees in one of the 2,899 case samples were grouped according to similarity of intersubject assessment score profiles by way of the hierarchical clustering procedure originally described by Ward (1963) and Ward and Hook (1963). This procedure begins by considering each individual as a unique cluster or subgroup, and then in successive steps combines subgroups until only one subgroup containing all individuals remains. At each step of the clustering process, two subgroups are merged in order to minimize intracluster variation while maximizing intercluster variation. That is, the objective is to find at each stage those two subgroups whose merger gives the minimum increase in the total within-group error sum of squares. The within-group error sum of squares is the sum of Euclidean distances from each data point in the two combined clus ters to the mean vector of the combined clusters.

The Ward and Hook (1963) clustering procedure typically involves computing a matrix of intersubject similarity functions (e.g., $d^{2}$ ) and searching the matrix for the minimum similarity value for subgroup combinations. However, where there are many subjects, the matrix-scanning approach becomes unworkable, because most computers do not have sufficient memory to store the large similarity function matrix. Thus, in this study, where the objective was to cluster a very large sample, a hierarchical clustering procedure presented by Anderberg (1973, pp. 145-148) was used to circumvent the computer data storage problem. This procedure, which is computationally equivalent to the matrix-scanning approach typically used, imple- 
ments the Ward and Hook (1963) method by operating on raw profile scores and summary statistics stored in the computer's memory rather than on the actual matrix of similarity measures.

There are two problems inherent in the hierarchical clustering procedure. First, there is no unambiguous solution to the problem of when to stop clustering. Logically, the solution is related to the amount of increase in the within-subgroup variation relative to the between-subgroup variation. Clustering should be terminated when an unacceptable increase in withinsubgroup variation has occurred. However, the determination of what constitutes an unacceptable increase remains subjective. In this study, as in others (Pinto, 1970; Taylor, 1968), the rate of change in within-subgroup variation was plotted, and the number of subgroups retained for further analysis was established at the stage in clustering that preceded the first substantially large inflection in within-subgroup variation.

The second problem with hierarchical clustering is that once an individual is assigned to a subgroup, that person becomes locked into that subgroup; thus, the assignment of individuals to subgroups is usually less than optimal at the conclusion of the grouping (Ward, 1963). As an adjunct to the hierarchical clustering procedure, the subgroups identified in the hierarchical analysis were subjected to a convergent means cluster analysis (Anderberg, 1973, p. 163). In this procedure an individual's distance to his/her original subgroup centroid is compared to his/her distance to all other subgroup centroids. If the distance to the original subgroup is the minimum, no change is made. If the distance to another subgroup is the minimum, the individual is moved to that subgroup, and the original and new subgroup centroids are changed to reflect the move. The process is repeated until no additional moves are made.

In some clustering research, subjccts not fitting any subgroup well (in a minimum distance sense) or fitting two or more subgroups equally well were dropped from the analysis (e.g.,
Schoenfeldt, 1974; Taylor, 1968). This typically resulted in the elimination of $20 \%$ to $25 \%$ of the sample. A similar treatment of subjects was not considered in this study, since an important goal of the research was to compare two prediction models developed in the same sample. Were misfits to be eliminated in the development of one model, the same subjects should be eliminated in the development of the other model in order to allow a fair comparison of the models. Even though there may be a theoretical justification for eliminating misfits from the clusterbased model, no theoretical justification for eliminating these subjects from the linear model was apparent. Therefore, no subjects were eliminated in the development of either model. All employees were assigned to a subgroup.

Validation. After final subgroup membership was determined, the subgroups were crosstabulated against the two criteria. The relationship between the subgroups and the criteria was analyzed in three ways:

1. Chi-square was computed to measure the statistical significance of association;

2. The index lambda was computed as an estimate of the practical utility of the association between the variables (Hays, 1963). Lambda is a measure of the percentage reduction in the probability of error in predicting the criteria from the subgroups. The statistic varies from zero, when knowing subgroup membership results in no reduction in error of prediction, to 1.0, when knowing subgroup membership results in $100 \%$ reduction in error of prediction;

3. A percent of correct classification (hit rate) for predicting the criteria was calculated. This was the sum over subgroups of the number of people in the most frequently appearing criterion categories divided by the total available sample size.

Cross-validation. Two steps were involved in cross-validating the actuarial model. First, the 2,899 cross-validation sample subjects were as- 
signed to one of the subgroups identified in the validation sample by means of the minimum distance qualifier, $d^{2}$. The distance of each subject in the cross-validation sample to each validation sample subgroup centroid was calculated, and individuals were assigned to the subgroup to which their distance was the minimum. Second, the cross-validation subgroups were cross-tabulated against the two criteria. Chi-square and lambda were calculated as in the validation sample. The hit rate was the sum over subgroups of the number of people in the most frequently appearing criterion categories identified in the validation sample divided by the total available cross-validation sample size.

\section{Linear Model}

Discriminant analysis. In the validation sample a discriminant analysis was performed on employment status, using the 13 analysis variables as predictor variables. Discriminant variable weights and a constant computed for each criterion category were used in conjunction with the a priori probability of criterion category membership to develop a predicted employment status for each individual. The significance of the discriminant analysis was tested by Mahalanobis $\mathrm{D}^{2}$. In addition, a hit rate was computed as the number of classifications, where predicted employment status was identical to actual em. ployment status divided by the total number of classifications.

In the cross-validation sample the discriminant weights computed in the validation sample were used to develop a predicted employment status and hit rate in the same manner as in the validation sample.

Regression analysis. In the validation sample the performance score was regressed on the $\mathbf{1 3}$ analysis variables. The resulting multiple correlation was tested for statistical significance, and the regression weights were used to calculate a predicted performance score for each sample member. Both the predicted and actual scores were dichotomized at their respective means and cross-tabulated against one another. Lambda was computed, and the hit rate was calculated as the number of comparisons where the predicted and actual criterion categories were the same, divided by the total number of comparisons.

In the cross-validation sample, predicted performance scores were computed by applying the validation sample regression weights to the 13 analysis variable scores. A cross-validated multiple correlation was calculated, and the performance scores were dichotomized and cross-tabulated. As for the validation sample, lambda and the hit rate were then computed.

\section{Results}

\section{Subgroup Development}

In the hierarchical reduction of the subgroups, a dramatic increase in within-subgroup variation occurred when 12 subgroups were merged into 11 subgroups, indicating that two very dissimilar subgroups had been merged. Therefore, 12 subgroups were retained for processing by the convergent means clustering technique. Application of this program resulted in a $10.7 \%$ reduction in the within-subgroup variation calculated for the 12 subgroups in the hierarchical analysis. The 12 revised subgroups were designated the validation sample subgroups in subsequent analyses.

Finally, based on the minimum distance qualifier $\left(d^{2}\right)$, the 2,899 cross-validation subjects were assigned to the subgroup they most closely resembled. Figure 1 presents the analysis variable profiles for three of the subgroups developed in the validation and cross-validation samples.' The 13 analysis variables used in the subgroup development are arrayed on the horizontal axis in the order presented in the Method section. The vertical axis represents the score range for each analysis variable, and the plotted points are the analysis variable means for the cluster.

'To conserve space, only three subgroup profiles are presen. ted. However, these profiles are representative of the correspondence between all validation and cross-validation profiles. 
Figure 1

Selected Analysis Variable Profiles for the Employee Subgroups:

Validation and Cross-Validation Samples

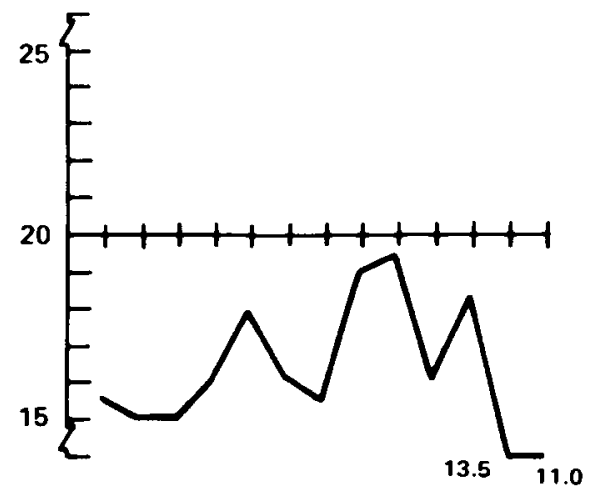

Subgroup 6

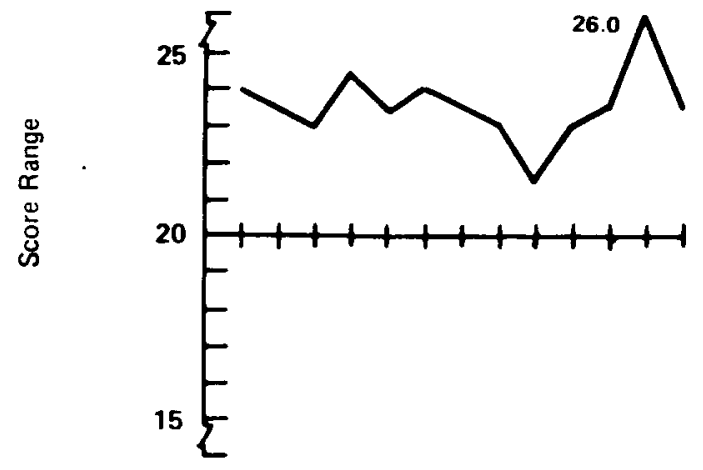

Subgroup 7

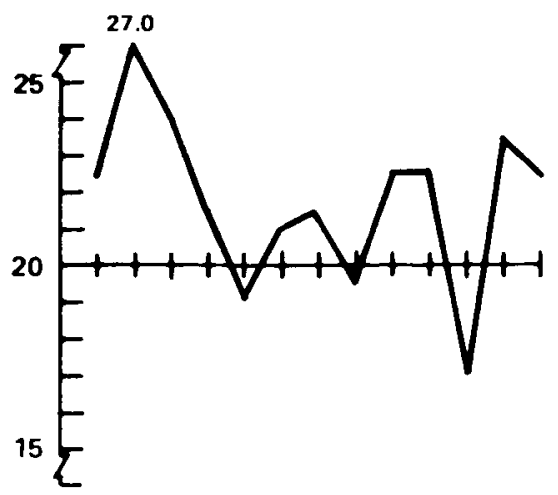

Subgroup 8
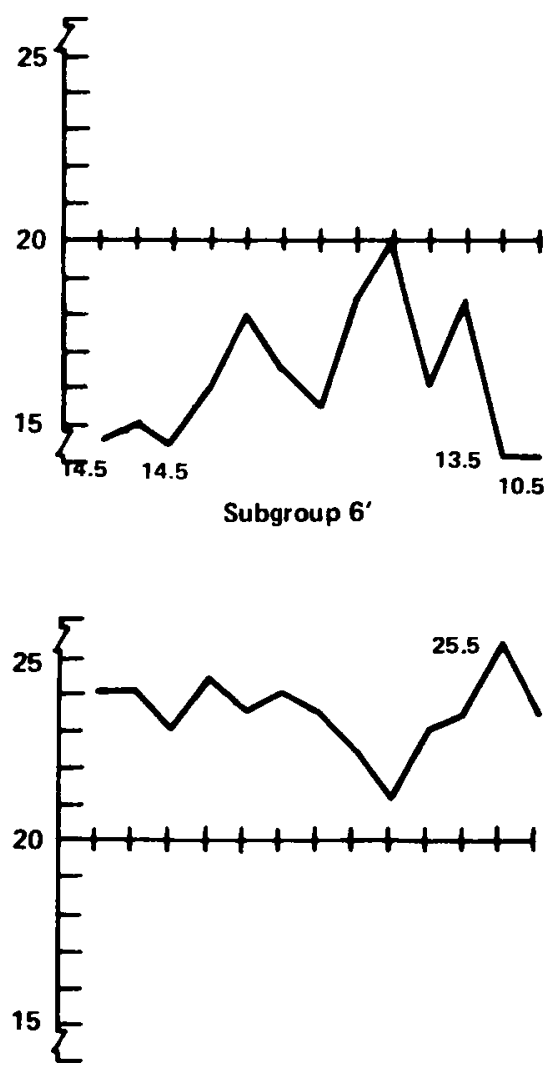

Subgroup 7'

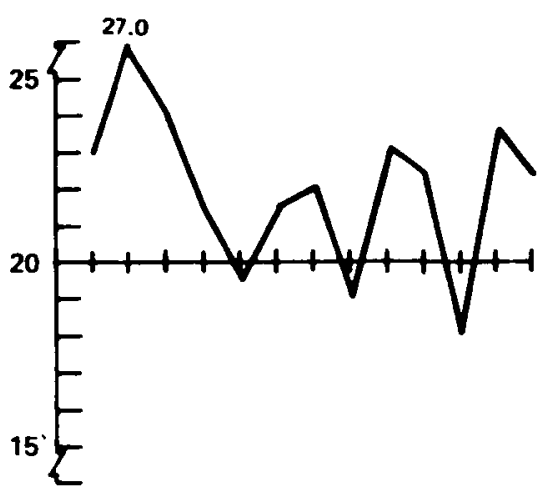

Subgroup 8'

Analysis Variables 
A visual comparison shows that the cross-validation profiles almost perfectly replicated the validation sample profiles. Moreover, for all subgroups the differences in mean values of the 13 analysis variables between the validation and cross-validation samples were extremely small, deviating by no more than one-tenth standard deviation between samples. The number of subjects in each subgroup differed from sample to sample, but by less than $2 \%$ of the total sample.

\section{Actuarial Model Prediction}

Employment status. The chi-square test of the association between the 12 employee subgroups and employment status, shown in Table 1 , yielded values of $71.14(p<.001)$ and 84.44 $(p<.001)$ in the validation and cross-validation samples, respectively. Lambda values of .00 and .01 indicated that knowing subgroup membership would reduce the probability of error in prediction by no more than $1 \%$. Despite statis- tical significance, knowledge of subgroup membership was not practically useful for predicting employment status. Reference to Table 1 shows that the reason for this was the high base rates $(67 \%, 68 \%)$ of the "With Company/Retired" employment status category in the two samples. Therefore, prediction of employment status was the same for all subgroups and yielded hit rates equal to the base rate of the With Company/ $\mathrm{Re}$ tired group in each sample.

Job performance. Table 2 presents the crosstabulation of subgroup membership and job performance for both samples. Chi-squares of $216.95(p<.001)$ and $252.25(p<.001)$ showed the association to be highly significant. Lambda indicated that knowing subgroup membership would result in a $26 \%$ and $25 \%$ reduction in the probability of error in predicting job performance. Hit rates of $64 \%$ and $63 \%$ showed that differential prediction based on subgroup membership would result in $12 \%$ to $13 \%$ more correct predictions than predicting by base rate alone

Table 1

Homogeneous Employee Subgroup by Employment Status

\begin{tabular}{|c|c|c|c|c|c|c|c|c|c|c|}
\hline \multirow[b]{4}{*}{ Subgroup } & \multicolumn{10}{|c|}{ Employment Status } \\
\hline & \multicolumn{5}{|c|}{ Validation Sample } & \multicolumn{5}{|c|}{ Cross-Validation Sample } \\
\hline & \multicolumn{3}{|c|}{$\begin{array}{c}\text { With Company/ } \\
\text { Retired }\end{array}$} & \multicolumn{2}{|c|}{$\begin{array}{l}\text { Not with } \\
\text { Company }\end{array}$} & \multicolumn{2}{|c|}{$\begin{array}{c}\text { With Company/ } \\
\text { Retired }\end{array}$} & \multicolumn{3}{|c|}{$\begin{array}{l}\text { Not With } \\
\text { Company }\end{array}$} \\
\hline & $\mathrm{f}$ & Row & 8 & $\mathrm{f}$ & Row 8 & $\mathbf{f}$ & Row 8 & $\mathrm{f}$ & Row & $\overline{8}$ \\
\hline 1 & $86 *$ & 53 & & 77 & 47 & $98 *$ & 57 & 74 & 43 & \\
\hline 2 & 98 * & 61 & & 62 & 39 & 98 * & 58 & 71 & 42 & \\
\hline 3 & $119 *$ & 69 & & 54 & 31 & $114 *$ & 66 & 60 & 34 & \\
\hline 4 & $175 *$ & 71 & & 72 & 29 & $207 *$ & 74 & 73 & 26 & \\
\hline 5 & 118 * & 50 & & 117 & 50 & $86 *$ & 47 & 99 & 53 & \\
\hline 6 & 125 * & 65 & & 67 & 35 & $116^{*}$ & 69 & 52 & 31 & \\
\hline 7 & $215 *$ & 78 & & 61 & 22 & 204 * & 78 & 57 & 22 & \\
\hline 8 & $146 *$ & 68 & & 70 & 32 & 164 * & 70 & 69 & 30 & \\
\hline 9 & $170 *$ & 66 & & 87 & 34 & 160 * & 65 & 85 & 35 & \\
\hline 10 & 153 * & 72 & & 59 & 28 & 152 * & 75 & 52 & 25 & \\
\hline 11 & $145^{\star}$ & 68 & & 68 & 32 & $151 *$ & 75 & 50 & 25 & \\
\hline 12 & $165 *$ & 74 & & 59 & 26 & 156 * & 70 & 67 & 30 & \\
\hline Total & 1714 & 67 & & 853 & 33 & $706 *$ & 68 & 809 & 32 & \\
\hline
\end{tabular}

*Validation Sample: The most frequently occuring criterion category for this subgroup; Cross-validation Sample: The criterion category the subgroup members were predicted to occupy. 
Table 2

Homogeneous Employee Subgroup by Job Performance

\begin{tabular}{|c|c|c|c|c|c|c|c|c|}
\hline \multirow[b]{4}{*}{ Subgroup } & \multicolumn{8}{|c|}{ Job Performance } \\
\hline & \multicolumn{4}{|c|}{ Validation Sample } & \multicolumn{3}{|c|}{ Cross-Validation } & Sample \\
\hline & Below & Mean & Above & Mean & Below & Mean & Above & Mean \\
\hline & $\mathbf{f}$ & Row $q$ & $\mathbf{f}$ & Row 8 & f & Row $q$ & $\mathrm{f}$ & Row $q$ \\
\hline 1 & 88 * & 67 & 44 & 33 & $102 *$ & 67 & 50 & 33 \\
\hline 2 & 67 & 49 & $69 *$ & 51 & 78 & 53 & $68 *$ & 47 \\
\hline 3 & $95 *$ & 59 & 66 & 41 & $113 *$ & 68 & 54 & 32 \\
\hline 4 & 127 * & 58 & 91 & 42 & $130 *$ & 50 & 128 & 50 \\
\hline 5 & 117 * & 60 & 77 & 40 & $99 *$ & 61 & 63 & 39 \\
\hline 6 & 147 * & 83 & 30 & 17 & $129 *$ & 86 & 21 & 14 \\
\hline 7 & 54 & 27 & 149 * & 73 & 43 & 21 & $160 *$ & 79 \\
\hline 8 & 49 & 29 & 117 * & 71 & 50 & 28 & $131 *$ & 72 \\
\hline 9 & 81 & 39 & $127 *$ & 61 & 83 & 43 & $112 *$ & 57 \\
\hline 10 & 72 & 40 & $107 *$ & 60 & 72 & 39 & 111 * & 61 \\
\hline 11 & 63 & 37 & $109 *$ & 63 & 63 & 37 & $108 *$ & 63 \\
\hline 12 & 81 & 42 & $113 *$ & 58 & 91 & 47 & $104 *$ & 53 \\
\hline Total & 1041 & 49 & 1049 & 51 & 1053 & 49 & 1111 & 51 \\
\hline
\end{tabular}

*Validation Sample: The most frequently occuring criterion category for the subgroup; Cross-validation Sample: The criterion category the subgroup members were predicted to occupy.

-an improvement of approximately $25 \%$ over the $51 \%$ base rate.

\section{Linear Model Prediction}

Employment status. The discriminant analysis for employment status yielded a Mahalanobis $\mathrm{D}^{2}$ of $115.00(p<.001)$, indicating that the 13 predictor variable means differed significantly between the With Company/Retired and Not With
Company categories. The classification tables computed on the samples are presented in Table 3. Hit rates of $67 \%$ and $68 \%$ were equal to the base rate of the With Company/Retired category. As with the actuarial model, despite a strong statistical association between the variables, no prediction of employment status could be made that was superior to the base rate prediction.

Table 3

Classification Rates for Predicted Employment Status Categories

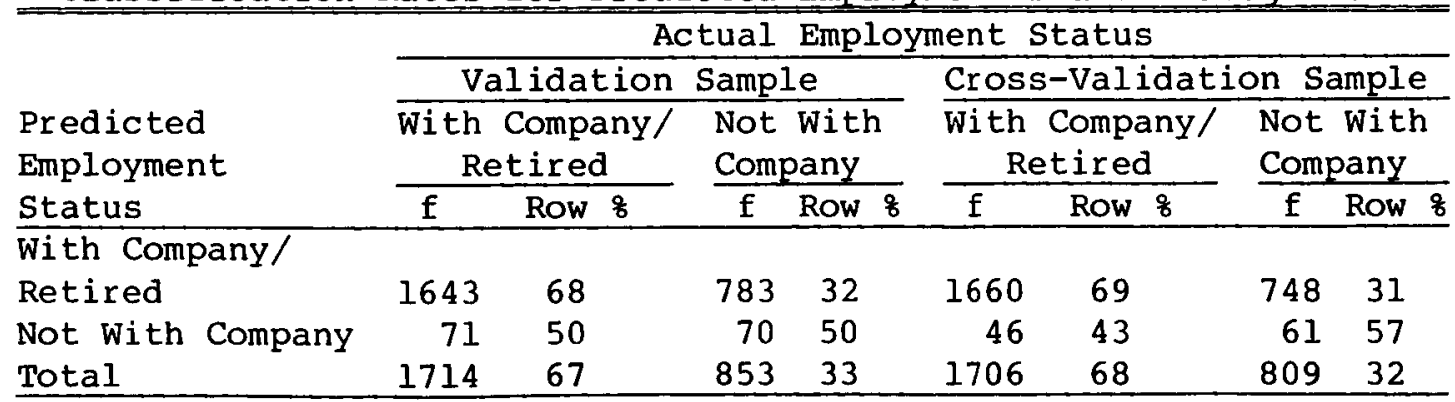


Table 4

Comparison of Predicted and Actual Job Performance

Actual Job Performance

\begin{tabular}{|c|c|c|c|c|c|c|c|c|c|}
\hline \multirow{3}{*}{$\begin{array}{l}\text { Predicted } \\
\text { Job } \\
\text { Performance }\end{array}$} & \multicolumn{4}{|c|}{ Validation Sample } & \multicolumn{5}{|c|}{ Cross-Validation Sample } \\
\hline & \multicolumn{2}{|c|}{ Below Mean } & \multicolumn{2}{|c|}{ Above Mean } & \multicolumn{3}{|c|}{ Below Mean } & \multicolumn{2}{|c|}{ Above Mean } \\
\hline & f & Row 8 & $\mathrm{f}$ & Row 8 & $\mathbf{f}$ & Row & $\overline{8}$ & $\mathrm{f}$ & Row \\
\hline bove Mean & 368 & 35 & 689 & 65 & 328 & 31 & & 736 & 69 \\
\hline elow Mean & 673 & 62 & 410 & 38 & 725 & 66 & & 375 & 34 \\
\hline otal & 1041 & 49 & 1099 & 51 & 1053 & 49 & & 1111 & 51 \\
\hline
\end{tabular}

Job performance. A multiple correlation of $.45(p<.001)$ resulted from the regression of job performance on the 13 analysis variables. The correlation of predicted scores, based on the validation sample regression weights, and actual scores in the holdout sample yielded a cross-validated multiple correlation of $.45 \quad(p<.001)$. Table 4 presents the cross-tabulations of the dichotomized predicted and actual scores for both samples. Lambda indicated that knowing predicted score group would result in a $25 \%$ and $33 \%$ reduction in the probability of error in predicting actual performance. Hit rates of $64 \%$ and $68 \%$ were calculated.

The improvement in efficiency of prediction on cross-validation, as indicated by the higher lambda and hit rate, was not expected, since the multiple correlation did not increase on crossvalidation. It was hypothesized that this improvement was an artifact resulting from the coarse grouping of job performance. Subsequent analyses supported this hypothesis. In each sample actual and predicted job performance were divided into five categories with equal frequencies and were cross-tabulated. The improvement in hit rate on cross-validation was $.7 \%$. Thus, although the increase did not vanish, it was substantially reduced. In addition, the 12 cross-validation subgroups were cross-tabulated with the five-category breakdown of the performance measure. The difference in hit rates between the actuarial and linear model predictions was . $5 \%$, indicating the two models were essentially equivalent in accuracy of prediction.

\section{Discussion}

The results have shown (1) that it is possible to identify homogeneous subgroups of people, given a heterogeneous sample of individuals profiled on various individual difference measures; (2) that these subgroups are significantly related to various external criteria; and (3) that the actuarial and linear models were equally effective in predicting these criteria. The finding that the models were equal in predictive accuracy suggests that the linear model can develop predictions as accurate as a model which accounts for unique configurations of individuals and their relationship to complex criteria. Thus, any decision to select one model over the other for operational use must be made on grounds other than predictive accuracy.

There are at least two reasons that argue for adoption of the actuarial model for operational use. First, it is very efficient (Owens, 1971, 1976). Assuming that the subgroups are significantly related to many external criteria, once a person's subgroup membership is determined, predictions can be made for all of these criteria. Compare this to the linear model that requires the generation of a regression or discriminant equation for each criterion and the subsequent development of an expectancy chart or classification table for each variable for ease of interpretation.

Second, and perhaps most important, use of the actuarial model promotes a better understanding of human behavior (Owens, 1971, 1976). Being a member of one subgroup rather 
than another has a unique meaning, represented by the subgroup's profile of test scores and the criteria that are significantly associated with subgroup membership.

For the sake of example, compare Subgroups 6 and 7. First, each subgroup has a distinct profile of analysis variable means. The profiles define the essential character of the subgroups, since the subgroups were developed on the basis of profile similarity. The constructs measured by the analysis variables provide a qualitative insight into what it means to be a subgroup member. Subgroup 6 is below average in intelligence and academic achievement. Its members had many negative family experiences. They have poor self-concepts and lack confidence in themselves. These individuals tend to be rigid and authoritarian in dealing with their employees. On the other hand, Subgroup 7 is above average in intelligence and academic achievement. Subgroup members had many positive family experiences and have a positive self-concept, with confidence in themselves and conviction in their ideas. They tend to be participative and flexible with their subordinates and believe in advancement due to ability. Second, the subgroups differ in terms of the external criteria associated with subgroup membership. Subgroup 6 is below average in job performance and Subgroup 7 is above average in job performance. Additionally, although the base rate problem proscribes an attrition prediction for either subgroup, some evidence of differential organizational longevity exists. Approximately $67 \%$ of Subgroup 6 is in the With Company/Retired category-a percentage equal to the total sample base rate. Approximately $78 \%$ of Subgroup 7 is in this category $-\mathrm{a}$ percentage significantly higher $(t=$ $5.42, p<.001$ ) than the $67 \%$ base rate. ${ }^{2}$

Thus, a better understanding of subgroup membership may be initially derived from information provided by the subgroups' unique

\footnotetext{
${ }^{2}$ It is beyond the scope of this paper to present a detailed description of each subgroup. However, subgroup profiles, analysis variable means, and a qualitative description of each subgroup are available from the author.
}

analysis variable profiles. Following this, an understanding of what it means to be a member of a particular subgroup may be developed by analyzing the subgroup-criterion relationships over many criteria; and since a basic assumption of the actuarial model is that the characteristics of the subgroup can be attributed to each member, the model provides for the description of individual behavior in terms of all criteria associated with subgroup membership.

Compare this to the linear model. If one were interested in predicting several criteria, a separate regression equation or discriminant analysis would have to be developed for each criterion. For each regression or discriminant analysis, the differential weighting of the predictor variables might provide some insight into the nature of the predictor-criterion relationship; however, the same predictor variables might have weights differing in sign and/or magnitude, depending on the criterion. Therefore, any understanding of the predictor-criterion relationship is in terms of the regression or discriminant weights for a specific criterion-not several criteria and not the individuals whose scores are being analyzed. On the other hand, the actuarial model provides for a greater understanding of human behavior by allowing the development of a descriptive and behavioral taxonomy based on stable, homogeneous subgroups of individuals.

\section{References}

Anderberg, M. R. Cluster analysis for applications. New York: Academic Press, 1973.

Dunnette, M. D. A modified model for test validation and selection research. Journal of Applied Psychology, 1963, 47, 317-323.

Frank, B. A. A comparison of an actuarial and a linear model for predicting managerial behavior. Unpublished doctoral dissertation, University of Houston, 1976.

Hays, W. L. Statistics. New York: Holt, Rinehart, \& Winston, 1963.

Lissitz, R. W., \& Schoenfeldt, L. F. Moderator subgroups for the estimation of educational performance: A comparison of prediction models. American Educational Research Journal, 1974, $11,63-75$. 
Owens, W. A. A quasi-actuarial basis for individual assessment. American Psychologist, 1971, 26, 992-999.

Owens, W. A. Background data. In M. Dunnette (Ed.), Handbook of industrial organization psychology. Chicago: Rand McNally, 1976.

Pinder, C. R., \& Pinto, P. R. Demographic correlates of managerial style. Personnel Psychology, 1974, 27, 257-270.

Pinto, P. R. Subgrouping in prediction: A comparison of moderator and actuarial approaches. Unpublished doctoral dissertation, University of Georgia, 1970.

Psychological Corporation. Advanced Personnel Test. New York: Author, 1970.

Richardson, Bellows, Henry, \& Co. The RBH Test of Nonverbal Reasoning. Washington, DC: Author, 1963.

Richardson, Bellows, Henry \& Co. Manager profile record. Washington, DC: Author, 1973.

Schoenfeldt, L. F. Utilization of manpower: Development and evaluation of an assessment-classification model for matching individuals with jobs. Journal of Applied Psychology, 1974, 59, 583-595.

Sines, J. O. Actuarial methods in personality assessment. In B. A. Maher (Ed.), Progress in experimental personality research (Vol. 3). New York: Academic Press, 1966.

Taylor, L. R. A quasi-actuarial approach to assessment. Unpublished doctoral dissertation, Purdue University, 1968.

Toops, H. A. The use of addends in experimental control, social census, and managerial research. Psychological Bulletin, 1948, 45, 41-74.
Toops, H. A. A research utopia in industrial psychology. Personnel Psychology, 1959, 12, 189-226.

Tyler, L. E. Toward a workable psychology of individuality. American Psychologist, 1959, 14, 75-81.

Vicino, F. L., \& Bass, B. M. Lifespace variables and managerial success. Journal of Applied Psychology. 1978, 63, 81-88.

Ward, J. H. Hierarchical grouping to optimize an objective function. Journal of the American Statistical Association, 1963, 58, 236-244.

Ward, J. H., Jr., \& Hook, M. E. Application of an hierarchical grouping procedure to a problem of grouping profiles. Educational and Psychological Measurement, 1963, 23, 69-81.

Wiggins, J. S. Personality and prediction: Principles of personality assessment. Reading, MA: Addison-Wesley, 1973.

\section{Acknowledgments}

This research was conducted and submitted in partial fulfillment of the requirements for the doctor of philosophy degree at the University of Houston. Thanks are accorded to Dr. H.G. Osburn. University of Houston, and Mr. C.P. Sparks, Exxon Co., U.S.A., for their assistance and guidance in completing this project.

\section{Author's Address}

Send requests for reprints or further information to Blake A. Frank, Personnel Research Department, The Southland Corporation, 2828 N. Haskell, Dallas, TX 75221. 